ARTICLE

https://doi.org/10.1038/s41467-019-08472-y

\title{
Large orbital polarization in nickelate-cuprate heterostructures by dimensional control of oxygen coordination
}

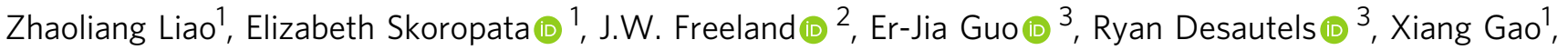 \\ Changhee Sohn', Ankur Rastogi ${ }^{1}$, T. Zac Ward (10 1, Tao Zou (id ${ }^{3}$, Timothy Charlton ${ }^{3}$, Michael R. Fitzsimmons ${ }^{3,4} \&$ \\ Ho Nyung Lee (1) ${ }^{1}$
}

Artificial heterostructures composed of dissimilar transition metal oxides provide unprecedented opportunities to create remarkable physical phenomena. Here, we report a means to deliberately control the orbital polarization in $\mathrm{LaNiO}_{3}(\mathrm{LNO})$ through interfacing with $\mathrm{SrCuO}_{2}(\mathrm{SCO})$, which has an infinite-layer structure for $\mathrm{CuO}_{2}$. Dimensional control of SCO results in a planar-type ( $\mathrm{P}-\mathrm{SCO}$ ) to chain-type (C-SCO) structure transition depending on the $\mathrm{SCO}$ thickness. This transition is exploited to induce either a $\mathrm{NiO}_{5}$ pyramidal or a $\mathrm{NiO}_{6}$ octahedral structure at the SCO/LNO interface. Consequently, a large change in the $\mathrm{Ni}$ $d$ orbital occupation up to $\sim 30 \%$ is achieved in P-SCO/LNO superlattices, whereas the $\mathrm{Ni} e_{\mathrm{g}}$ orbital splitting is negligible in C-SCO/LNO superlattices. The engineered oxygen coordination triggers a metal-to-insulator transition in SCO/LNO superlattices. Our results demonstrate that interfacial oxygen coordination engineering provides an effective means to manipulate the orbital configuration and associated physical properties, paving a pathway towards the advancement of oxide electronics.

\footnotetext{
${ }^{1}$ Materials Science and Technology Division, Oak Ridge National Laboratory, Oak Ridge, TN 37831, United States. ${ }^{2}$ Advanced Photon Source, Argonne National Laboratory, Argonne, IL 60439, United States. ${ }^{3}$ Neutron Scattering Division, Oak Ridge National Laboratory, Oak Ridge, TN 37831, United States. ${ }^{4}$ Department of Physics and Astronomy, University of Tennessee, Knoxville, TN 37996, United States. Correspondence and requests for materials should be addressed to H.N.L. (email: hnlee@ornl.gov)
} 
T he correlated $d$-orbital plays a central role in creating collective phenomena in transition metal oxides, since the $s$-electrons of transition metals are transferred to oxygen ions, and the remaining $d$-electrons determine the delicate interplay between spin, charge, and orbital degrees of freedom ${ }^{1}$. For example, superexchange can induce either antiferromagnetic or ferromagnetic coupling of spins depending on the orbital filling and ordering, yielding a vast array of magnetic ground states $^{2-5}$. In high transition temperature $\left(T_{\mathrm{C}}\right)$ cuprate superconductors, the $x^{2}-y^{2}$ orbital character is crucial for Cooper pairing ${ }^{6}$. The remarkable Mott metal-insulator transition often found in correlated oxides can be engineered by tailoring the orbital occupancy ${ }^{7}$. Thus, identifying ways to deliberately control the orbital degree of freedom in correlated oxides is highly desirable to discover electronic and magnetic phenomena that are useful for developing oxide electronic and spintronic devices. Interface engineering is a powerful method to manipulate such orbital degrees of freedom ${ }^{7-13}$ and has revealed many intriguing phenomena not possible in bulk material counterparts, such as interfacial charge transfer, orbital covalency, and broken inversion symmetry at nanometer scale ${ }^{12-19}$.

Rare-earth nickelate $\left(\mathrm{RNiO}_{3}\right)$ heterostructures provide good experimental and theoretical frameworks to investigate influence of orbital occupation on the physical properties ${ }^{17-24}$. The $d^{7}$ ionic configuration of $\mathrm{Ni}^{3+}$ in nickelates contains a full $t_{2 \mathrm{~g}}$ shell and a single electron occupying the $e_{\mathrm{g}}$ orbital. This orbital configuration generally favors a Jahn-Teller distortion as observed in $\mathrm{LaMnO}_{3}{ }^{25}$. However, the $e_{\mathrm{g}}$ orbitals in nickelates are doubly degenerate. A recent theoretical study proposed that the planar $x^{2}-y^{2}$ orbital order could be realized by spatially confining $\mathrm{LaNiO}_{3}$ into a ultrathin layer between two insulators, leading to a cuprate-like Fermi surface and, subsequently, high $T_{\mathrm{C}}$ superconductivity 23,24 . This idea triggered enormous experimental efforts to study the $\mathrm{RNiO}_{3}$ systems. However the orbital polarization caused by spatial confinement and strain was much smaller than the predicted one ${ }^{17,26-28}$. Resonant inelastic X-ray scattering indicated a very minor $e_{\mathrm{g}}$ splitting energy $\left(\Delta E_{e_{\mathrm{g}}}\right)$ induced by heterostructural engineering 29 . It has also been suggested that nickelate thin films prefer to retain their preferred electronic and structural configurations determined by the bulk phase, which limits the control of the orbital parameters through heterostructural engineering ${ }^{30}$.

Here, we report that one can largely manipulate the occupancy of $\mathrm{Ni} 3 d$ orbitals in $\mathrm{LNO}^{-} \mathrm{SrCuO}_{2}$ (SCO) heterostructures by dimensional control of the SCO layers. The SCO which in the bulk phase is orthorhombic $(\mathrm{Cmcm})$ with a double-chain structure formed in a perovskite like framework with missing apical oxygen in thin films ${ }^{31,32}$. Upon reducing the thickness when grown in thin films, the planar-type structure of $\mathrm{SCO}(\mathrm{P}-\mathrm{SCO})$ is transformed to a chain-type structure (C-SCO $)^{33-35}$. The $\mathrm{CuO}_{2}$ planes of $\mathrm{P}-\mathrm{SCO}$ and $\mathrm{C}-\mathrm{SCO}$ are parallel and perpendicular with respect to the film plane, respectively. This difference effectively yields two different heterostructures (see Fig. 1a, b). In the case of a $\mathrm{P}-\mathrm{SCO} / \mathrm{LNO}$ heterostructure grown on a $\mathrm{B}$-site terminated $\mathrm{ABO}_{3}$ perovskite substrate, the interfacial atomic stacking sequence is hypothesized to be $\left(\mathrm{CuO}_{2} / \mathrm{Sr}\right) /\left(\mathrm{NiO}_{2} / \mathrm{LaO}\right)$, leading to a truncated $\mathrm{NiO}_{5}$ pyramid structure (see Fig. 1a). On the other hand, the stacking sequence for the C-SCO/LNO interface would become $(\mathrm{CuO} / \mathrm{SrO}) /\left(\mathrm{NiO}_{2} / \mathrm{LaO}\right)$, which yields an octahedrally coordinated $\mathrm{Ni}$ and thus no modification of the LNO structure (see Fig. 1b). Our results demonstrate how the unique infinitelayer structure offered in SCO can be utilized to induce a very large Ni $3 d$ orbital polarization in $\mathrm{P}-\mathrm{SCO} / \mathrm{LNO}$ superlattices or to create degenerate (i.e., little or no polarization) $\mathrm{Ni} e_{\mathrm{g}}$ orbitals in $\mathrm{C}-\mathrm{SCO} / \mathrm{LNO}$ superlattices as sketched in Fig. 1a, b. The observed changes in the electronic and orbital states may have a profound impact on transport properties of SCO/LNO superlattices.

\section{Results}

Dimensional control of the sub-lattice structure of $\mathrm{SrCuO}_{2}$. LNO and SCO films and their superlattices were grown on $\mathrm{TiO}_{2}$ terminated (001) $\mathrm{SrTiO}_{3}$ (STO) substrates by pulsed laser epitaxy. The growth condition was optimized to achieve high-quality SCO films in the bulk planar structure (see Supplementary Fig. 1), and all films were atomically flat with step-terrace features on their surfaces (See Supplementary Fig. 2). As shown in Fig. 1c, X-ray diffraction (XRD) $\theta-2 \theta$ scans of an 80 unit-cell (u.c.) SCO film revealed clearly the SCO 002 peak near $2 \theta=53^{\circ}$, confirming the bulk-like infinite-layer structure ${ }^{33,34}$. Using the same growth conditions, high-quality LNO films were obtained as well.
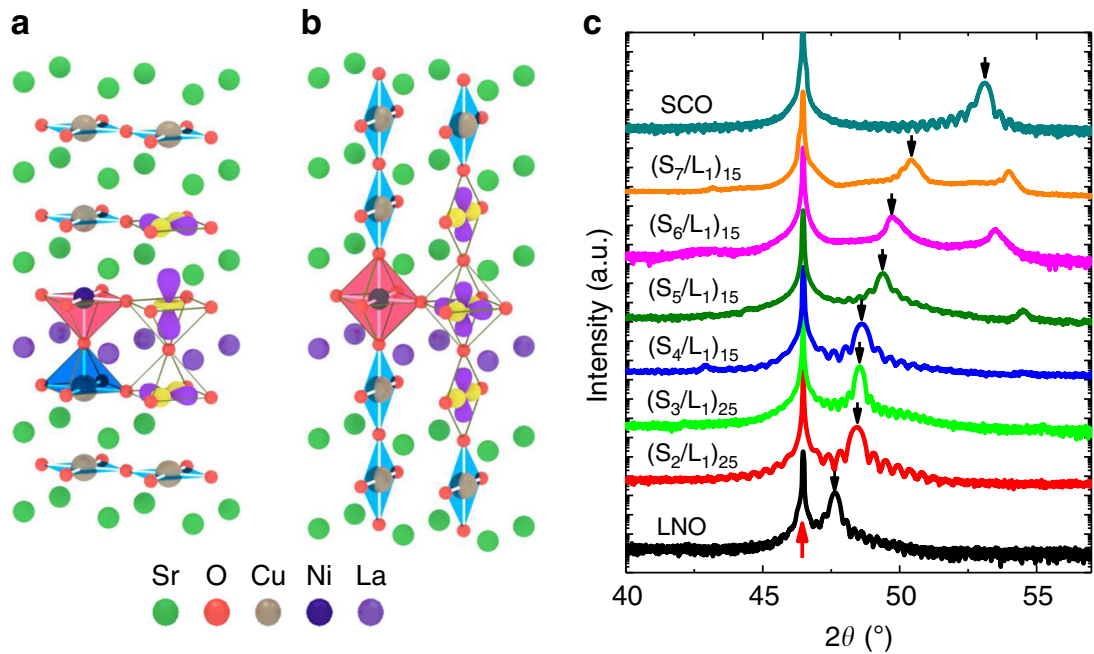

Fig. 1 Interfacial engineering of oxygen coordination in $\mathrm{SrCuO}_{2} / \mathrm{LaNiO}_{3}$ superlattices. Schematics for $\mathrm{SrCuO}_{2} / \mathrm{LaNiO} \mathrm{H}_{3}$ (SCO/LNO) heterostructures with a planar-type $\mathrm{SrCuO}_{2}$ and $\mathbf{b}$ chain-type $\mathrm{SrCuO}_{2}$, resulting in different oxygen coordination and orbital polarization of $\mathrm{Ni}$ ions in $\mathrm{LaNiO}$. $\mathbf{c}$ X-ray diffraction (XRD) $\theta-2 \theta$ scans of a $\mathrm{LaNiO}_{3}$ film (34 nm in thickness), $\left(\left(\mathrm{SrCuO}_{2}\right)_{N} /\left(\mathrm{LaNiO}_{3}\right)_{1}\right)_{M}$ (noted as $\left.\left(\mathrm{S}_{N} / \mathrm{L}_{1}\right)_{M}\right)$ superlattices, and a $\mathrm{SrCuO} 2$ film $(28 \mathrm{~nm})$. The red and black arrows indicate the $\mathrm{SrTiO}_{3} 002$ peak and the peaks from superlattices or films, respectively, showing a clear modulation of out-of-plane lattice constants 
When growing $\left(\mathrm{SCO}_{N} / \mathrm{LNO}_{1}\right)_{M}$ superlattices (noted as $\left.\mathrm{S}_{N} / \mathrm{L}_{1}\right)$, a systematic change in XRD spectra was observed with increasing the SCO layer thickness. Since the P-SCO and C-SCO have quite different out-of-plane lattice constants of $\sim 3.45 \AA\left(2 \theta=53^{\circ}\right)$ and $\sim 3.8 \AA\left(2 \theta=47.8^{\circ}\right)$, respectively ${ }^{33,35}$, the position of the main superlattice peak SL(0) can be used to identify the SCO structure type. For relatively thick SCO layers (e.g., $N=7$ u.c.), $\mathrm{SL}(0)$ was located between the SCO 002 and the LNO 002 XRD peaks, suggesting the formation of the planar-type SCO structure within the superlattice. When the SCO layer thickness is reduced to $2 \mathrm{u}$. c., the SL $(0)$ peak shifts close to the LNO 002 peak, indicating that 2 u.c.-thick SCO has a similar out-of-plane lattice constant with that of LNO. This result suggests that a chain-type SCO structure was formed in the $S_{2} / L_{1}$ superlattice. With increasing the SCO film thickness to 5 u.c., a sudden large shift of the superlattice peaks occurred. This shift suggests the critical thickness of the phase conversion from $\mathrm{P}-\mathrm{SCO}$ to $\mathrm{C}-\mathrm{SCO}$ to be at 5 u.c.. This thickness-driven transition of the SCO structure in SCO/LNO superlattices was also observed for other LNO sublayer thicknesses (see Supplementary Fig. 3).

Further evidence of the structural transition triggered by reducing the SCO thickness was observed from XRD reciprocal space mapping (RSM). The RSM shown in Fig. 2a indicates that all the superlattices and reference SCO and LNO films were coherently grown on STO substrates. The reciprocal lattice vector $\left(q_{\mathrm{z}}\right)$ of thick SCO superlattices (e.g., $N=7$ and 16 in $\mathrm{S}_{N} / \mathrm{L}_{1}$ ) was very close to that of a $\mathrm{P}-\mathrm{SCO}$ film, confirming that the SCO layers in the superlattices possess the bulk-like planar-type structure. On the other hand, the $q_{\mathrm{z}}$ of thin SCO superlattices $(N=2-4)$ was similar to that of a LNO film. Interestingly, as shown in Fig. $2 \mathrm{~b}$, the SCO thickness dependent change in the out-of-plane lattice constant $\left(c_{\mathrm{SL}}\right)$ calculated from the RSMs around the 103 peak $\left(c_{\mathrm{SL}}=3 / q_{\mathrm{z}}\right)$ clearly reveals a sudden transition at around $5 \mathrm{u}$. c. of the SCO sublayer thickness. If the bulk-like $\mathrm{P}-\mathrm{SCO}$ is maintained when the SCO thickness is reduced, one can only expect a gradual increase in $c_{\mathrm{SL}}$. Therefore, our observation of the step-like transition shown in Fig. 2a, b unambiguously demonstrates that the planar-type lattice structure of SCO can be converted into the chain-type structure by reducing the SCO thickness below 5 u.c. Detailed XRD and transport analyses further suggest the formation of a twin domain structure within the chain-type SCO (see supplementary Fig. 4), originating from the two-fold symmetry of C-SCO on the four-fold (001) STO substrate.

Such a change in the orientation of the oxygen plane can be confirmed as a change in the orbital occupancy measured from linearly polarized X-ray absorption spectroscopy (XAS) acquired in fluorescence yield (FY) mode. The orbital occupancy of $\mathrm{Cu}$ $x^{2}-y^{2}$ and $3 z^{2}-r^{2}$ orbitals was probed by the XAS measured with in-plane $(\sigma)$ and out-of-plane $(\pi)$ polarized $x$-rays, respectively.

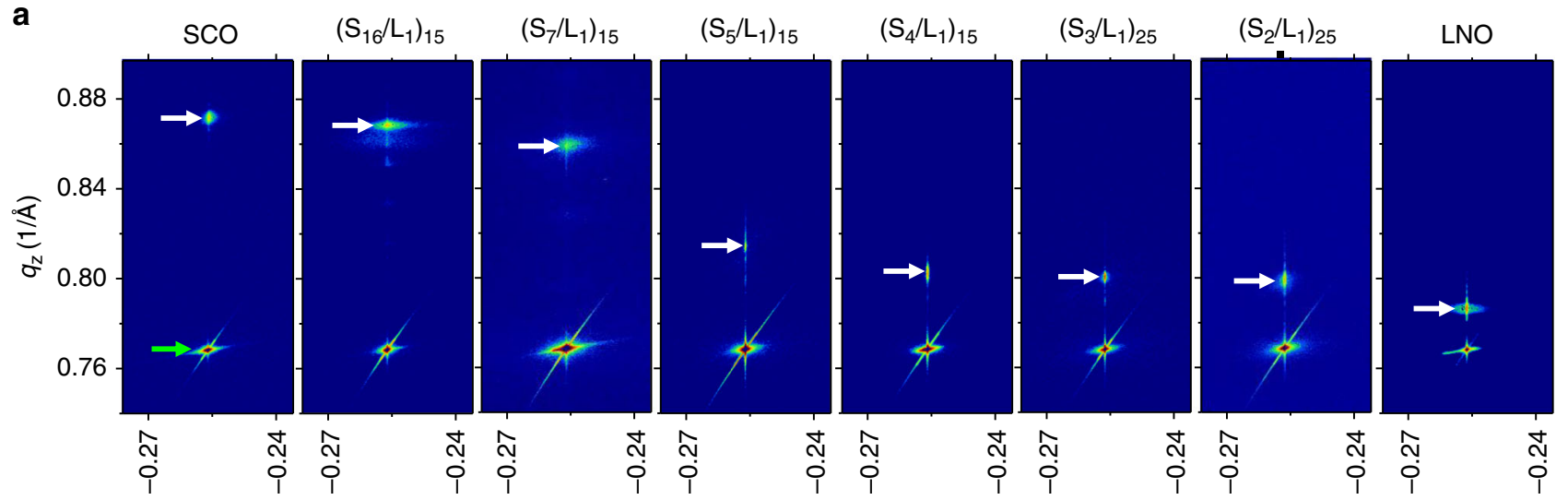

$q_{\mathrm{x}}(1 / \AA)$
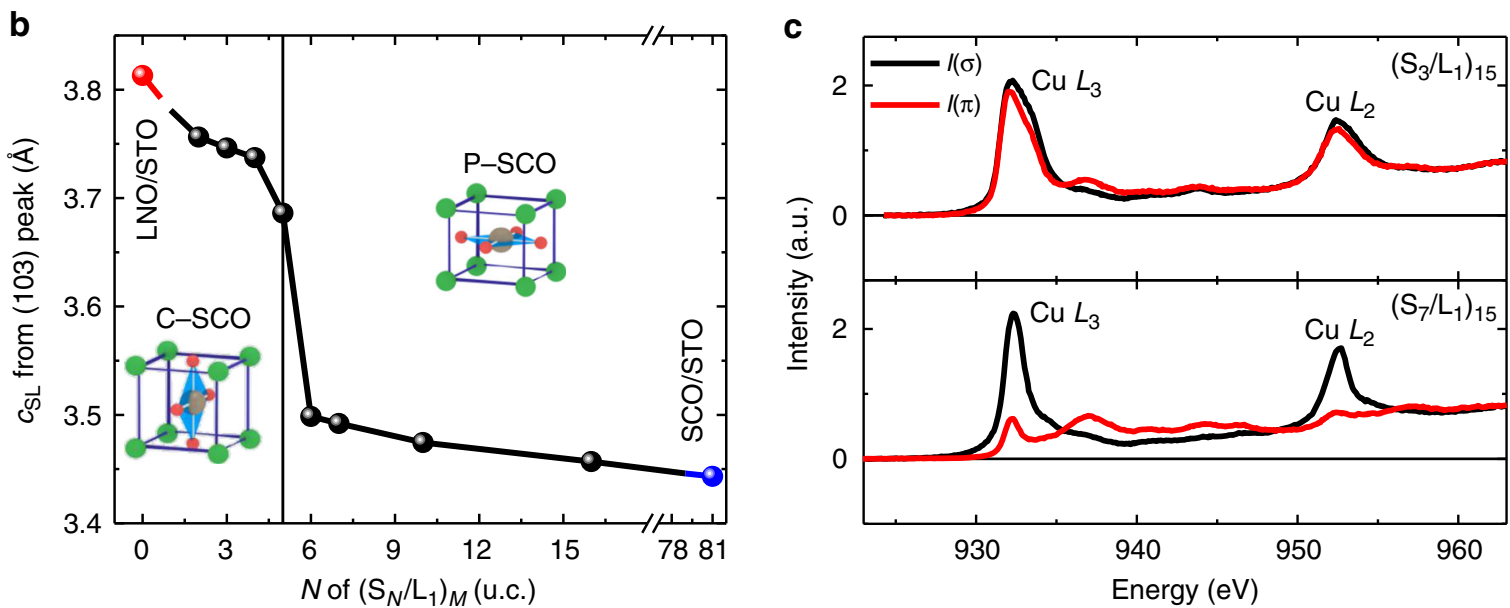

Fig. 2 Dimensional control of the sub-lattice structure of $\mathrm{SrCuO}_{2}$. a Reciprocal space mapping of the 103 peak of a $\mathrm{LaNiO}_{3}(\mathrm{LNO})$ film, $\left((\mathrm{SrCuO})_{N} / \mathrm{LNO}_{1}\right)_{M}$ superlattices and a $\mathrm{SrCuO}_{2}$ (SCO) film. The green and white arrows indicate $\mathrm{SrTiO}_{3}$ (STO) substrate and film/superlattice peaks, respectively. b Out-ofplane lattice constant $(c)$ as a function SCO sublayer thickness $(N)$. The sudden change of $c$ across $N=5$ u.c. indicates a structural transition from a planar type to a chain type. c Polarization dependent XAS of $\mathrm{Cu} L_{2,3}$ edge for $\mathrm{S}_{7} / \mathrm{L}_{1}$ (top panel) and $\mathrm{S}_{3} / \mathrm{L}_{1}$ (bottom panel) superlattices, demonstrating a clear contrast on the orbital population 
As shown in Fig. 2c, the large $I(\sigma)$ versus $I(\pi)$ XAS of the $\mathrm{Cu} L_{2,3^{-}}$ edge of the $S_{7} / L_{1}$ superlattice indicates exclusive $3 d_{x^{2}-y^{2}}$ character, consistent with orbital configuration in layer-structured cuprates (see supplementary Fig. 5). By contrast, $I(\sigma)$ and $I(\pi)$ share a similar intensity and shape for the $S_{3} / L_{1}$ superlattice, suggesting the chain-type SCO structure and $3 d_{y^{2}-z^{2}}$ orbital character ${ }^{36}$. Additionally, the change in the SCO microstructure and oxygen coordination of $\mathrm{Ni}$ were confirmed by scanning transmission electron microscopy (STEM) (see Supplementary Fig. 6), consistent with XRD and XAS data.

Engineered $\mathrm{Ni}$ orbital polarization in $\mathrm{SrCuO}_{2}-\mathrm{LaNiO}_{3}$ superlattices. Now we turn to investigate the reconstruction of the $\mathrm{Ni}$ $e_{g}$ orbital. Figure 3 a shows the XAS of the Ni $L_{2}$-edge of an $\mathrm{S}_{7} / \mathrm{L}_{1}$ superlattice. Since the $\mathrm{Ni} L_{3}$-edge largely overlaps with the La $M$ edge, and FY of the $\mathrm{La}^{3+}\left(4 f^{0}\right) M$-edge deviates from the true XAS for the conventional detection geometry used, the $\mathrm{Ni} L_{2}$-edge is more suitable to illustrate the orbital reconstruction (see supplementary Fig. 7$)^{17}$. The $I(\sigma)$ exhibits a higher intensity than $I(\pi)$, indicating that holes prefer to occupy the $3 d_{x^{2}-y^{2}}$ orbital. For the $\mathrm{S}_{3} / \mathrm{L}_{1}$ superlattice, the $I(\sigma)$ and $I(\pi)$ intensities are similar to each other. Therefore, the Ni $e_{\mathrm{g}}$ orbital is degenerate (Fig. 3b). The different orbital configuration between the $S_{7} / L_{1}$ superlattice and the $S_{3} / L_{1}$ superlattice is also observed in total electron yield mode (see supplementary Fig. 7), indicating that the observed polarization dependence of the FY XAS originates from the intrinsic orbital polarization. The orbital configuration in the $\mathrm{S}_{3} / \mathrm{L}_{1}$ superlattice confirms the chain-type SCO structure within the $S_{3} / L_{1}$ superlattice, which recovers the octahedral coordination. Using the difference between in-plane polarized absorption $I(a b)$ and out-of-plane polarized absorption $I(c)$, the ratio of unoccupied states, $r$, can be quantified using the following sum rule 17,37

$$
r=\frac{h_{3 z^{2}-r^{2}}}{h_{x^{2}-y^{2}}}=\frac{3 I(c)}{4 I(a b)-I(c)}
$$

Here, $a, b$, and $c$ are the Cartesian coordination axis of the substrates with $c$ along out-of-plane direction and $h$ is the hole occupancy. The $\pi$-polarized absorption $I(\pi)$ was corrected for the angle $\theta=20^{\circ}$ between the incident X-rays and sample plane in our experimental setup using $I(c)=\left[I(\pi)-I(a b) \sin ^{2} \theta\right] /$ $\cos ^{2} \theta$, while the orientation of the incident beam with respect to the sample did not affect $I(\sigma)$ and $I(a b)=I(\sigma)$. The linear dichroism (LD) $[L D=I(c)-I(a b)]$ shown in Fig. 3a, b further emphasizes the orbital polarization of the $S_{7} / L_{1}$ superlattice. Using the integrated intensity of $I(a b)$ and $I(c)$ from backgroundsubtracted XAS, the hole ratio $r$ can be quantified (see supplementary Fig. 8). The $r$ values for $\mathrm{S}_{7} / \mathrm{L}_{1}$ and $\mathrm{S}_{3} / \mathrm{L}_{1}$ superlattices are 0.7 and 1.0, respectively, suggesting a predominant $x^{2}-y^{2}$ hole character in $\mathrm{S}_{7} / \mathrm{L}_{1}$ and degenerate orbital in $\mathrm{S}_{3} / \mathrm{L}_{1}$. Previously, the $r$ value of the largest orbital polarization $\left[P=\left(n_{x^{2}-y^{2}}-n_{3 z^{2}-r^{2}}\right) /\left(n_{x^{2}-y^{2}}+n_{3 z^{2}-r^{2}}\right)\right]$ achieved by inducing tensile strain was $\sim 1.19^{26}$. The spatial confinement in LAO/LNO superlattices has been reported to result in a value of $r \sim 1.05^{17,27}$. The hole ratio that we observed cannot be compared directly to these previous values since our experiments had a flipping orbital configuration in which the $3 d_{3 z^{2}-r^{2}}$ orbital has more hole occupancy. The relative change of occupancy $(\equiv|1-r|)$ is instead used here for a quantitative comparison. In comparison to $\sim 19 \%$ by strain $^{26}$ and $\sim 5 \%$ by spatial confinement 17,27 , the observed $30 \%$ change of $\mathrm{Ni}$ orbital occupancy in $\mathrm{P}-\mathrm{SCO} / \mathrm{LNO}$ superlattice confirms the significant impact of oxygen coordination on orbital reconstruction.

The prevailing $3 d_{x^{2}-y^{2}}$ character cannot be explained by spatial confinement, which favors the $3 d_{3 z^{2}-r^{2}}$ hole occupancy due to the confinement of the $3 d_{3 z^{2}-r^{2}}$ orbital in the $z$ direction $17,23,24,38$. We attribute the observed large orbital polarization in the $S_{7} / L_{1}$ superlattice to the $\mathrm{NiO}_{5}$ pyramid structure, which is expected to lower the $3 d_{3 z^{2}-r^{2}}$ with respect to $3 d_{x^{2}-y^{2}} 39$. In fact, our polarization dependent XAS revealed quite similar features to the square-planar $\mathrm{La}_{4} \mathrm{Ni}_{3} \mathrm{O}_{8}$ and $\mathrm{YBa}_{2} \mathrm{Cu}_{3} \mathrm{O}_{7}$ (YBCO) single crystals ${ }^{36,40}$. The $\mathrm{La}_{4} \mathrm{Ni}_{3} \mathrm{O}_{8}$ is comprised of $\mathrm{NiO}_{2}$ planar structures while the main building block of $\mathrm{YBCO}$ is a $\mathrm{CuO}_{5}$ pyramid $^{40,41}$. These two materials possess similar $e_{\mathrm{g}}$ band structures with the $3 d_{3 z^{2}-r^{2}}$ lying lower than $3 d_{x^{2}-y^{2}}$. In sharp contrast, there is a negligible orbital polarization when the apical oxygen is retained in the $\mathrm{S}_{3} / \mathrm{L}_{1}$ superlattice. Although there is still tensile strain on $\mathrm{LNO}$ within the $\mathrm{S}_{3} / \mathrm{L}_{1}$ superlattice, the observed degenerate $e_{\mathrm{g}}$ orbital indicates that the octahedrally coordinated $\mathrm{Ni}$ prefers to retain a bulk-like electronic configuration. This retainment of bulk orbital configuration was reported previously $^{30}$ and is also observed in our LNO films (see Supplementary Fig. 9). The corresponding $e_{\mathrm{g}}$ orbital energy
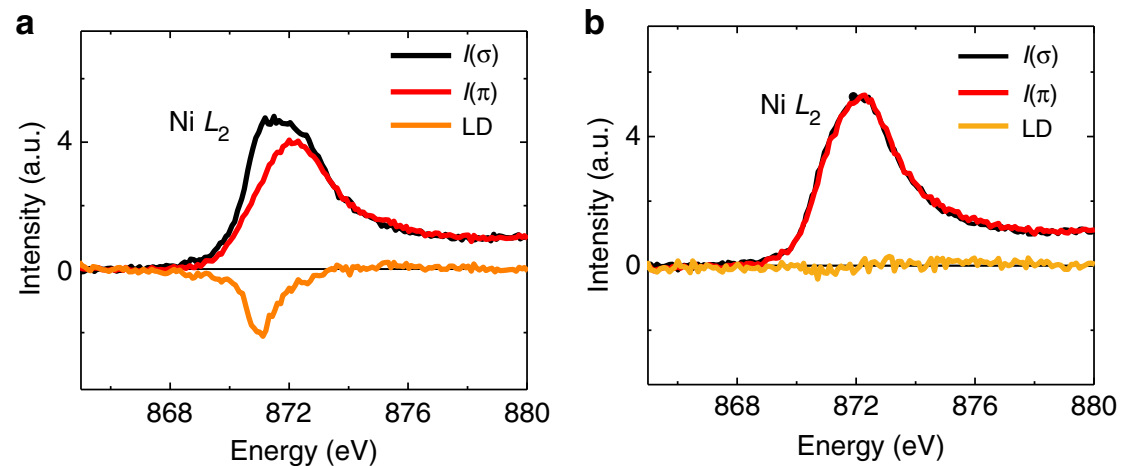

C

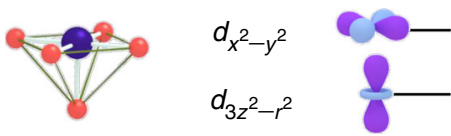

d
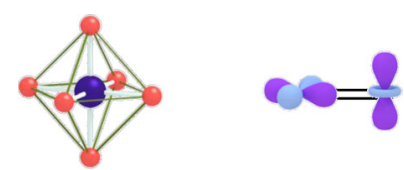

Fig. 3 Orbital polarization of the Ni-3d orbital by oxygen coordination control. a Polarization dependent X-ray absorption spectroscopy (XAS) of Ni $L_{2}$ edge for $\mathbf{a} S_{7} / L_{1}$ and $\mathbf{b} S_{3} / L_{1}$ superlattices. Large linear dichroism (LD) is observed for the $S_{7} / L_{1} S L$, whereas the LD is nearly zero for the $S_{3} / L_{1} S L$. Schematics of the $e_{g}$ orbital configuration are shown for $\mathbf{c} \mathrm{S}_{7} / \mathrm{L}_{1}$ and $\mathbf{d} \mathrm{S}_{3} / \mathrm{L}_{1}$ superlattices 
a

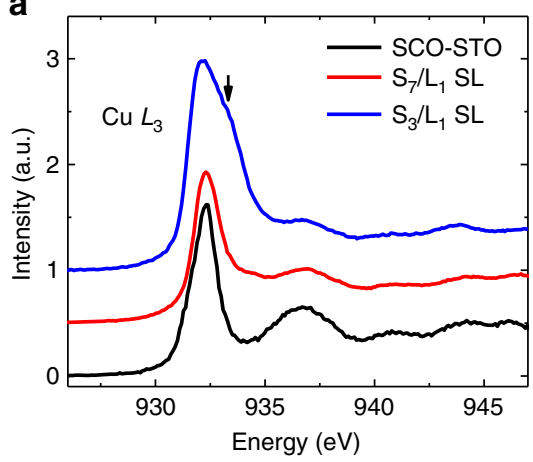

b

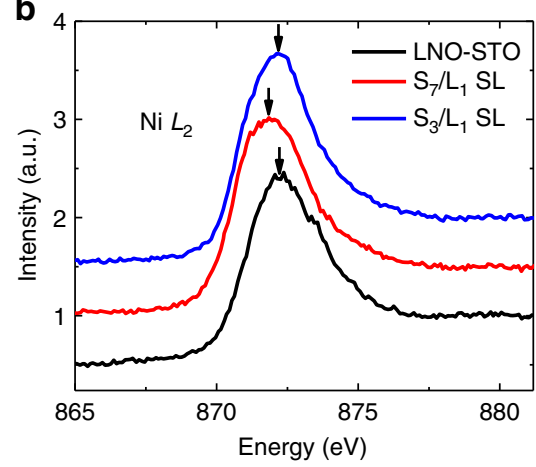

Fig. 4 The charge states of $\mathrm{Cu}$ and $\mathrm{Ni}$ in different superlattices. a X-ray absorption spectroscopy (XAS) of $\mathrm{Cu} \mathrm{L}_{3}$-edge for a $\mathrm{SrCuO}_{2}$ (SCO) film and $\left(\mathrm{SCO}_{7} /\left(\mathrm{LaNiO}_{3}\right)_{1}\right.$ ) (noted as $\mathrm{S}_{7} / \mathrm{L}_{1}$ ) and $\mathrm{S}_{3} / \mathrm{L}_{1}$ superlattices $(\mathrm{SL})$. A shoulder indicated by the black arrow arises from Cu $\mathrm{d}^{9} \underline{\mathrm{L}}$ state. $\mathbf{b} \times A S$ of $\mathrm{Ni} \mathrm{L}_{2}$-edge for a $\mathrm{LaNiO}_{3}$ (LNO) film and $\mathrm{S}_{7} / \mathrm{L}_{1}$ and $\mathrm{S}_{3} / \mathrm{L}_{1}$ superlattices. A slight shift of the peak position to a lower energy for $\mathrm{S}_{7} / \mathrm{L}_{1}$ superlattice suggests an electron doping in the LNO layer. The XAS is obtained by averaging linearly polarized XAS $(I=(I(\sigma)+I(\pi)) / 2)$

diagrams for the $\mathrm{NiO}_{5}$ pyramid and the $\mathrm{NiO}_{6}$ octahedron are schematically plotted in Fig. $3 \mathrm{c}\left(\mathrm{S}_{7} / \mathrm{L}_{1}\right)$ and Fig. $3 \mathrm{~d}\left(\mathrm{~S}_{3} / \mathrm{L}_{1}\right)$. Using the outermost filled orbital, a sketch of the orbital configuration in SCO/LNO superlattice is plotted Fig. 1a, b, demonstrating the designed orbital configuration through heterostructural engineering.

The charge states of $\mathrm{Cu}$ and $\mathrm{Ni}$ at interfaces. The charge states of $\mathrm{Cu}$ and $\mathrm{Ni}$ are further investigated using XAS. As shown in Fig. 4 a, the $\mathrm{Cu} L$-edge spectrum of a $\mathrm{S}_{7} / \mathrm{L}_{1}$ superlattice shows a characteristic $\mathrm{Cu}^{2+}$ feature as observed in SCO films ${ }^{36,42}$. Meanwhile, the $S_{3} / L_{1}$ superlattice shows a strong shoulder at $\sim 933.28 \mathrm{eV}$, which is positioned $\sim 1 \mathrm{eV}$ higher than that of the $\mathrm{Cu}^{2+}$ peak and is consistent with the $\mathrm{d}^{9} \underline{L}$ state ${ }^{36,42}$. Here $\underline{\mathrm{L}}$ represents the ligand hole. The doped holes are unlikely from interfacial charge transfer, since the $\mathrm{Ni}$ in $\mathrm{S}_{3} / \mathrm{L}_{1}$ maintains $\mathrm{Ni}^{3+}$ as demonstrated by the lack of $\mathrm{Ni} L$-edge shift (see Fig. $4 \mathrm{~b}$ ). Further, $\mathrm{La}$ interdiffusion should favor $\mathrm{Cu}^{1+}$. It is more likely that the hole arises from oxygen excess $\mathrm{SrCuO}_{2+x}$. For the $\mathrm{S}_{7} / \mathrm{L}_{1}$ superlattice, the Ni $L$-edge shifts by about $-0.3 \mathrm{eV}$ relative to that of the LNO film. Since a $1 \mathrm{eV}$ shift corresponds to roughly one electron valence change ${ }^{43,44}$, the energy shift suggests an averaged $\mathrm{Ni}^{2} .7+$ valence state in $\mathrm{S}_{7} / \mathrm{L}_{1}$. It has been reported that doped electrons at a $\mathrm{LNO} / \mathrm{La}_{2} \mathrm{CuO}_{4}$ interface are predominantly accommodated by change of $\mathrm{Ni}$ valence ${ }^{45}$. A similar situation may occur for a SCO/LNO interface where a possible formation of oxygen vacancies may dope the interface state with electrons.

Controlled metal-to-insulator transition. Functionally, we see that these observed changes to electronic and orbital states have a profound impact on transport properties of SCO/LNO superlattices. Figure $5 \mathrm{a}$ shows the transport properties of $\mathrm{S}_{N} / \mathrm{L}_{1}$ superlattices with different $\mathrm{SCO}$ thicknesses. The $\mathrm{S}_{N} / \mathrm{L}_{1}$ superlattices exhibit a metallic behavior $(\mathrm{d} R / \mathrm{d} T>0)$ to insulating behavior $(\mathrm{d} R / \mathrm{d} T<0)$ transition $(\mathrm{MIT})$ with increasing the SCO layer thickness. The critical thickness for MIT is 5 u.c., which coincides with the critical thickness for a structural transition (see Fig. 2). This fact suggests that the $\mathrm{P}-\mathrm{SCO}_{N} / \mathrm{LNO}_{1}$ and $\mathrm{C}-\mathrm{SCO}_{N} /$ $\mathrm{LNO}_{1}$ superlattices are insulating and metallic, respectively. Since LNO will undergo a MIT with reducing thickness across 3-5 u.c. ${ }^{46-49}$, the single unit-cell LNO should be insulating and thus the metallic behavior arises from the C-SCO layer, which can be explained by the hole doping as observed by XAS (see Fig. 4a).

Figure $5 \mathrm{~b}, \mathrm{c}$ show the LNO layer thickness dependent transport properties in $\mathrm{P}-\mathrm{SCO}_{7} / \mathrm{L}_{N}$ and $\mathrm{C}-\mathrm{SCO}_{3} / \mathrm{L}_{N}$ superlattices, respectively. With reducing thickness of $\mathrm{LNO}$ layers, the $\mathrm{P}-\mathrm{SCO}_{7} / \mathrm{L}_{N}$ superlattices gradually evolve from a metallic state to an insulating state. Such behavior is similar to MIT in single LNO films, which is associated with dimensional crossover ${ }^{46,49}$. What is more, the 3 u.c. critical thickness for MIT in $\mathrm{P}-\mathrm{SCO}_{7} / \mathrm{L}_{N}$ is also similar to bare LNO films ${ }^{46-49}$. These results strongly suggest that the MIT in $\mathrm{P}-\mathrm{SCO}_{7} / \mathrm{L}_{N}$ is driven by LNO sub-layers. Since the C-SCO is metallic, all $\mathrm{C}-\mathrm{SCO}_{3} / \mathrm{L}_{N}$ superlattices exhibit good metallic behavior regardless of the thickness of LNO (see Fig. 5c). However, there is still a sharp decrease of the resistance when the LNO thickness is reduced from $N=3$ to 2 u.c., while the $\mathrm{C}-\mathrm{SCO}_{3} / \mathrm{L}_{1}$ and $\mathrm{C}-\mathrm{SCO}_{3} / \mathrm{L}_{2}$ showed similar resistance values. This result implies a MIT in the LNO layers when reducing LNO thickness from 3 to 2 u.c. The different critical thickness $\left(t_{c}\right)$ for MIT of LNO in $\mathrm{P}-\mathrm{SCO}_{7} / \mathrm{L}_{N}\left(t_{\mathrm{c}}=3\right)$ and in $\mathrm{C}-\mathrm{SCO}_{3} / \mathrm{L}_{N}\left(t_{\mathrm{c}}=2\right)$ indicates that a MIT in 3 u.c. LNO sublayer occurs when the top surface of the LNO layer is transformed from the octahedral coordination to the pyramidal coordination (Fig. 5d, e). This result is further emphasized by comparing the resistance of $\mathrm{P}-\mathrm{SCO}_{7} / \mathrm{L}_{3}$ with $\mathrm{C}-\mathrm{SCO}_{7} / \mathrm{L}_{3}$ superlattices in Fig. 5f. Although the metallic C-SCO layer also contributes to the transport, the transport behavior of $\mathrm{C}-\mathrm{SCO}_{3} / \mathrm{L}_{3}$ is dominated by the LNO layer since the resistance of $\mathrm{C}-\mathrm{SCO}_{3} / \mathrm{L}_{1}$ is $\sim 2$ times larger than that of $\mathrm{C}-\mathrm{SCO}_{3} /$ $\mathrm{L}_{3}$. To further confirm the effect of oxygen coordination on transport, we measured the transport properties of $\mathrm{STO}_{7} / \mathrm{L}_{N}$ superlattices where the LNO interface has the same octahedral coordination as that in $\mathrm{C}-\mathrm{SCO}_{3} / \mathrm{L}_{3}$. The critical thickness for MIT in $\mathrm{STO}_{7} / \mathrm{L}_{N}$ is 2 u.c. (see Supplementary Fig. 10), which is the same with $\mathrm{C}-\mathrm{SCO}_{3} / \mathrm{L}_{N}$ superlattices and thinner than $\mathrm{P}-\mathrm{SCO}_{7} / \mathrm{L}_{N}$ superlattices. This indicates that recovering the top pyramid $\mathrm{NiO}_{5}$ in $\mathrm{P}-\mathrm{SCO}_{7} / \mathrm{L}_{3}$ to a full octahedral structure in $\mathrm{STO}_{7} / \mathrm{L}_{3}$ or $\mathrm{C}-\mathrm{SCO}_{3} / \mathrm{L}_{3}$ will drive the LNO layer to be metallic. As shown in Fig. $5 f$, the $\mathrm{STO}_{7} / \mathrm{L}_{3}$ is metallic and has a similar resistance with $\mathrm{C}-\mathrm{SCO}_{3} / \mathrm{L}_{3}$. The relative small change of resistance from $\mathrm{P}-\mathrm{SCO}_{7} /$ $\mathrm{L}_{3}$ to $\mathrm{STO}_{7} / \mathrm{L}_{3}$ is due to the crossover MIT in LNO and also the fact that P-SCO is not a good insulator and contributes to the transport as well. Higher LNO resistance in $\mathrm{P}-\mathrm{SCO} / \mathrm{LNO}$ superlattices could be due to the reduced oxygen coordination, as observed in bare LNO films ${ }^{50,51}$. The smaller Ni $e_{\mathrm{g}}$ bandwidth in pyramidal coordination and reduced in-plane electron hopping strength due to preferential $3 z^{2}-r^{2}$ orbital occupation would increase the resistance. Further, the appearance of $\mathrm{Ni}^{2+}$ may also play a role by opening a charge excitation gap ${ }^{38,43}$.

\section{Discussion}

In conclusion, we have demonstrated that the orbital polarization and transport properties of $\mathrm{SCO} / \mathrm{LNO}$ superlattices can be manipulated by templating the infinite structure of SCO into 
a
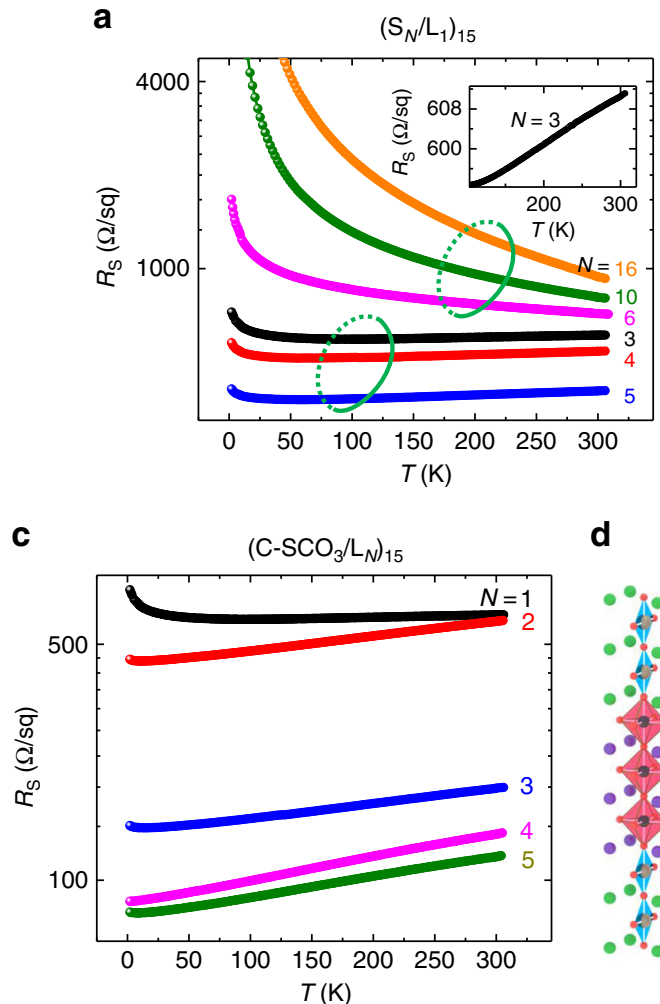

b

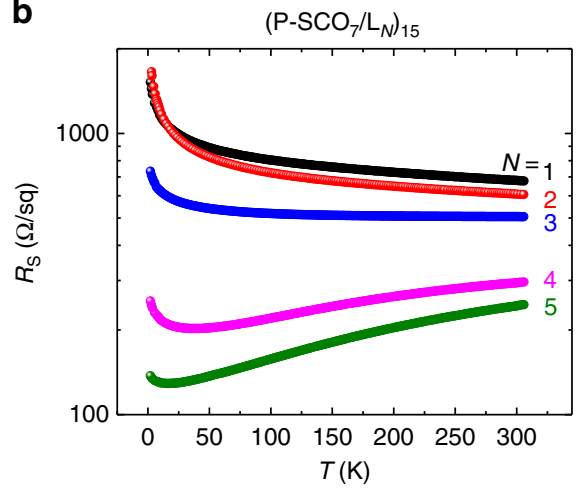

e

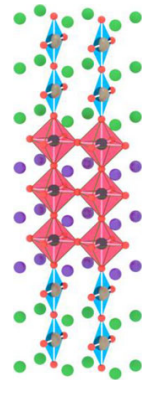

f

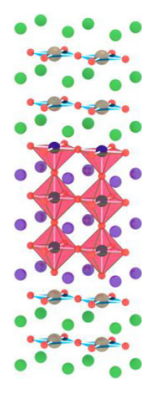

Fig. 5 Metal-to-insulator transition by dimensionality control in superlattices. Temperature dependent sheet resistance of a $\left(\mathrm{S}_{N} / \mathrm{L}_{1}\right)_{15}, \mathbf{b}\left(\mathrm{P}-\mathrm{SCO} \mathrm{O}_{7} / \mathrm{L}_{N}\right)_{15}$ and $\mathbf{c}\left(C-\mathrm{SCO}_{3} / \mathrm{L}_{N}\right)_{15}$ for various layer thickness $(N)$ of $\mathbf{a} \mathrm{SCO}$ or $\mathbf{b}, \mathbf{c} \mathrm{LNO}$. The green ellipses in a group separately the metallic and insulating superlattices. $\mathrm{S}, \mathrm{L}, \mathrm{P}-\mathrm{SCO}$, and $\mathrm{C}-\mathrm{SCO}$ represent $\mathrm{SrCuO}_{2}(\mathrm{SCO}), \mathrm{LaNiO}_{3}(\mathrm{LNO})$, planar-type $\mathrm{SCO}$, and chain-type $\mathrm{SCO}$, respectively. Structural models for $\mathbf{d}$ (C-SCO $/$ $\left.\mathrm{L}_{3}\right)_{15}$ and $\mathbf{e}\left(\mathrm{P}-\mathrm{SCO}_{3} / \mathrm{L}_{3}\right)_{15}$ superlattices. $\mathbf{f}$ Temperature dependent sheet resistance of $\left(\mathrm{C}-\mathrm{SCO}_{7} / \mathrm{L}_{3}\right)_{15}$ (blue curves), $\left(\mathrm{P}-\mathrm{SCO} \mathrm{CO}_{3} / \mathrm{L}_{3}\right)_{15}($ red curves), and $\left(\mathrm{STO}_{7} / \mathrm{L}_{3}\right)_{15}$ (black curves). The transport properties illustrate a metal-to-insulator transition in 3 u.c. LNO layer when the top surface is transformed from the octahedral coordination to the pyramidal coordination

LNO through dimensionally controlling the oxygen coordination. Reducing the SCO thickness below 5 u.c. resulted in a structural transition from a planar-type to a chain-type structure. At $\mathrm{SCO} /$ LNO interfaces, the planar-type SCO structure induced a $\mathrm{NiO}_{5}$ pyramidal structure in LNO, whereas the chain-type SCO structure yielded a $\mathrm{NiO}_{6}$ octahedron in $\mathrm{LNO}$, allowing us to selectively control the out-of-plane and in-plane orbital occupancies. This type of oxygen coordination manipulation could enhance the $\mathrm{Ni}$ orbital polarization up to $\sim 30 \%$. This enhancement is larger than that achieved from other means, including by strain $(\sim 19 \%)$ or spatial confinement $(\sim 5 \%)$. Our findings unambiguously demonstrate how the interfacial oxygen coordination engineering can effectively manipulate the orbital configuration and electronic properties, providing a possible new approach to realizing superconducting nickelates with high $T_{\mathrm{c}}$. The oxygen coordination control using $\mathrm{SCO}$ as a template could also be applied to other perovskites to explore previously inaccessible orbital-driven phenomena.

\section{Methods}

Sample growth. All superlattices and thin films were grown on $\mathrm{TiO}_{2}$ terminated (001) $\mathrm{SrTiO}_{3}$ substrates by pulsed laser epitaxy using a KrF excimer laser $(\lambda=248$ $n m)$. The single $\mathrm{TiO}_{2}$ terminated and atomically flat $\mathrm{SrTiO}_{3}$ substrates were obtained by the etching with standard buffered-HF and subsequent annealing at $950^{\circ} \mathrm{C}$ for $1.5 \mathrm{~h}$. The laser fluence and repetition rate were fixed at $2 \mathrm{~J} \mathrm{~cm}^{-2}$ and $5 \mathrm{~Hz}$, respectively. The oxygen partial pressure and substrate temperature were maintained at $100-150$ mTorr and $650^{\circ} \mathrm{C}$, respectively.

Sample characterization. The surface morphology was characterized by atomic force microscopy. The lattice structure was characterized by a high-resolution fourcircle X-ray diffractometer. Transport measurements were performed in a van-der-
Pauw geometry using a Physical Property Measurement System. The TEY and FY XAS measurements of the $\mathrm{Cu}$ and $\mathrm{Ni}$ edges were performed at the beamline 4-ID-C of the Advanced Photon Source. XAS was measured at $300 \mathrm{~K}$, with the $\mathrm{X}$-rays at a grazing $\left(20^{\circ}\right)$ angle to the films surface, and the TFY detector at $90^{\circ}$ from the incoming X-rays.

\section{Data availability}

The data that support the findings of this study are available from the corresponding author upon reasonable request.

Received: 23 April 2018 Accepted: 8 January 2019

Published online: 04 February 2019

\section{References}

1. Tokura, Y. \& Nagaosa, N. Orbital physics in transition-metal oxides. Science 288, 462-468 (2000).

2. Goodenough, J. B. Theory of the role of covalence in the perovskite-type manganites [La,M(II)] $\mathrm{MnO}_{3}$. Phys. Rev. 100, 564-573 (1955).

3. Goodenough, J. B. An interpretation of the magnetic properties of the perovskite-type mixed crystals $\mathrm{La}_{1-\mathrm{x}} \mathrm{Sr}_{\mathrm{x}} \mathrm{CoO}_{3-\lambda}$. J. Phys. Chem. Solids 6 , 287-297 (1958).

4. Kanamori, J. Superexchange interaction and symmetry properties of electron orbitals. J. Phys. Chem. Solids 10, 87-98 (1959).

5. Tokura, Y. \& Tomioka, Y. Colossal magnetoresistive manganites. J. Magn. Magn. Mater. 200, 1-23 (1999).

6. Keimer, B., Kivelson, S. A., Norman, M. R., Uchida, S. \& Zaanen, J. From quantum matter to high-temperature superconductivity in copper oxides. Nature 518, 179-186 (2015).

7. Aetukuri, N. B. et al. Control of the metal-insulator transition in vanadium dioxide by modifying orbital occupancy. Nat. Phys. 9, 661-666 (2013).

8. Hwang, H. Y. et al. Emergent phenomena at oxide interfaces. Nat. Mater. 11, 103-113 (2012). 
9. Ohshima, R. et al. Strong evidence for d-electron spin transport at room temperature at a $\mathrm{LaAlO}_{3} / \mathrm{SrTiO}_{3}$ interface. Nat. Mater. 16, 609-614 (2017).

10. Matsuno, J. et al. Interface-driven topological Hall effect in $\mathrm{SrRuO}_{3}-\mathrm{SrIrO}_{3}$ bilayer. Sci. Adv. 2, e1600304 (2017).

11. Chakhalian, J. et al. Orbital reconstruction and covalent bonding at an oxide interface. Science 318, 1114-1117 (2007).

12. Chakhalian, J. et al. Magnetism at the interface between ferromagnetic and superconducting oxides. Nat. Phys. 2, 244-248 (2006).

13. Okamoto, S. et al. Charge Transfer in Iridate-Manganite Superlattices. Nano. Lett. 17, 2126-2130 (2017).

14. Nichols, J. et al. Emerging magnetism and anomalous Hall effect in iridate-manganite heterostructures. Nat. Commun. 7, 12721 (2016).

15. Lee, H. N., Christen, H. M., Chisholm, M. F., Rouleau, C. M. \& Lowndes, D. H. Strong polarization enhancement in asymmetric threecomponent ferroelectric superlattices. Nature 433, 395-399 (2005).

16. Petrie, J. R. et al. Enhanced bifunctional oxygen catalysis in strained $\mathrm{LaNiO}_{3}$ perovskites. J. Am. Chem. Soc. 138, 2488-2491 (2016).

17. Benckiser, E. et al. Orbital reflectometry of oxide heterostructures. Nat. Mater. 10, 189-193 (2011)

18. Frano, A. et al. Orbital control of noncollinear magnetic order in nickel oxide heterostructures. Phys. Rev. Lett. 111, 106804 (2013).

19. Disa, A. S. et al. Orbital engineering in symmetry-breaking polar heterostructures. Phys. Rev. Lett. 114, 026801 (2015).

20. Gibert, M., Zubko, P., Scherwitzl, R., Íñiguez, J. \& Triscone, J.-M. Exchange bias in $\mathrm{LaNiO}_{3}-\mathrm{LaMnO}_{3}$ superlattices. Nat. Mater. 11, 195-198 (2012).

21. Grisolia, M. N. et al. Hybridization-controlled charge transfer and induced magnetism at correlated oxide interfaces. Nat. Phys. 12, 484-492 (2016).

22. van Veenendaal, M. Anomalous ground states at the interface between two transition-metal compounds. Phys. Rev. B 78, 165415 (2008).

23. Chaloupka, J. \& Khaliullin, G. Orbital order and possible superconductivity in $\mathrm{LaNiO}_{3} / \mathrm{LaMO}_{3}$ superlattices. Phys. Rev. Lett. 100, 016404 (2008).

24. Hansmann, P. et al. Turning a nickelate fermi surface into a cupratelike one through heterostructuring. Phys. Rev. Lett. 103, 016401 (2009).

25. Sawada, H., Morikawa, Y., Terakura, K. \& Hamada, N. Jahn-Teller distortion and magnetic structures in $\mathrm{LaMnO}_{3}$. Phys. Rev. B 56, 12154 (1997).

26. $\mathrm{Wu}, \mathrm{M}$. et al. Strain and composition dependence of orbital polarization in nickel oxide superlattices. Phys. Rev. B 88, 125124 (2013).

27. Freeland, J. W. et al. Orbital control in strained ultra-thin $\mathrm{LaNiO}_{3} / \mathrm{LaAlO}_{3}$ superlattices. Europhys. Lett. 96, 57004 (2011).

28. Disa, A. S., Walker, F. J., Ismail-Beigi, S. \& Ahn, C. H. Research update: orbital polarization in $\mathrm{LaNiO}_{3}$-based heterostructures. APL Mater. 3, 062303 (2015).

29. Fabbris, G. et al. Orbital engineering in nickelate heterostructures driven by anisotropic oxygen hybridization rather than orbital energy levels. Phys. Rev. Lett. 117, 147401 (2016).

30. Tung, I. C. et al. Connecting bulk symmetry and orbital polarization in strained $\mathrm{RNiO}_{3}$ ultrathin films. Phys. Rev. B 88, 205112 (2013).

31. Teske, C. L. \& Mueller-Buschbaum, H. Über Erdalkalimetall-Oxocuprate, V. Zur Kenntnis von $\mathrm{Ca}_{2} \mathrm{CuO}_{3}$ und $\mathrm{SrCuO}_{2}$. Z. Anorg. Allg. Chem. 379, 234-241 (1970).

32. Norton, D. P. et al. Superconductivity in $\mathrm{SrCuO}_{2}-\mathrm{BaCuO}_{2}$ superlattices: formation of artificially layered superconducting materials. Science $\mathbf{2 6 5}$, 2074-2077 (1994).

33. Zhong, Z. C., Koster, G. \& Kelly, P. J. Prediction of thickness limits of ideal polar ultrathin films. Phys. Rev. B 85, 121411(R) (2012).

34. Harter, J. W. et al. Doping evolution and polar surface reconstruction of the infinite-layer cuprate $\mathrm{Sr}_{1-x} \mathrm{La}_{\mathrm{x}} \mathrm{CuO}_{2}$. Phys. Rev. B 92, 035149 (2015).

35. Samal, D. et al. Experimental evidence for oxygen sublattice control in polar infinite layer $\mathrm{SrCuO}_{2}$. Phys. Rev. Lett. 111, 096102 (2013).

36. Hawthorn, D. G. et al. Resonant elastic soft $\mathrm{x}$-ray scattering in oxygen-ordered $\mathrm{YBa}_{2} \mathrm{Cu}_{3} \mathrm{O}_{6+\delta}$. Phys. Rev. B 84, 075125 (2011).

37. van der Laan, G. Sum rules and fundamental spectra of magnetic X-Ray dichroism in crystal field symmetry. J. Phys. Soc. Jpn. 63, 2393-2400 (1994).

38. Cao, Y. W. et al. Engineered Mott ground state in a $\mathrm{LaTiO}_{3} / \mathrm{LaNiO}_{3}$ heterostructure. Nat. Commun. 7, 10418 (2015).

39. Chancey, C. C. \& O'Brien, M. C. M. The Jahn-Teller effect: An introduction and current review. Am. J. Phys. 61, 688-697 (1993).

40. Zhang, J. J. et al. Large orbital polarization in a metallic square-planar nickelate. Nat. Phys. 13, 864-869 (2017).

41. Izumi, F. et al. Crystal structure of the orthorhombic form of $\mathrm{Ba}_{2} \mathrm{YCu}_{3} \mathrm{O}_{7-\mathrm{x}}$ at 42 K. Jpn. J. Appl. Phys. 26, L1193-L1196 (1987).
42. Chen, C. T. et al. Out-of-plane orbital characters of intrinsic and doped holes in $\mathrm{La}_{2-\mathrm{x}} \mathrm{Sr}_{\mathrm{x}} \mathrm{CuO}_{4}$. Phys. Rev. Lett. 68, 2543 (1992).

43. Guo, E. J. et al. Oxygen diode formed in nickelate heterostructures by chemical potential mismatch. Adv. Mater. 30, 1705904 (2018).

44. Liu, J. et al. Effect of polar discontinuity on the growth of superlattices. Appl. Phys. Lett. 96, 133111 (2010).

45. Wrobel, F. et al. Digital modulation of the nickel valence state in a cupratenickelate heterostructure. Phys. Rev. Mater. 2, 035001 (2018).

46. Scherwitzl, R. et al. Metal-insulator transition in ultrathin $\mathrm{LaNiO}_{3}$ Films. Phys. Rev. Lett. 106, 246403 (2011).

47. Boris, A. V. et al. Dimensionality control of electronic phase transitions in nickel-oxide superlattices. Science 332, 937-940 (2011).

48. King, P. D. C. et al. Atomic-scale control of competing electronic phases in ultrathin $\mathrm{LaNiO}_{3}$. Nat. Nanotechnol. 9, 443-447 (2014).

49. Sakai, E. et al. Gradual localization of $\mathrm{Ni} 3 \mathrm{~d}$ states in $\mathrm{LaNiO}_{3}$ ultrathin films induced by dimensional crossover. Phys. Rev. B 87, 075132 (2013).

50. Kumah, D. P. et al. Effect of surface termination on the electronic properties of $\mathrm{LaNiO}_{3}$ films. Phys. Rev. Appl. 2, 054004 (2014).

51. Kumah, D. P. et al. Tuning the structure of nickelates to achieve twodimensional electron conduction. Adv. Mater. 26, 1935 (2014)

\section{Acknowledgements}

This work was supported by the U.S. Department of Energy (DOE), Office of Science, Basic Energy Sciences (BES), Materials Sciences and Engineering Division. Use of the Advanced Photon Source, an Office of Science User Facility operated for the U.S. DOE, Office of Science by Argonne National Laboratory, was supported by the U.S. DOE under Grant No. DEAC02-06CH11357. Part of XAS measurements and experimental assistance for thin film synthesis by E.G., R.D., T.Z., T.C. and M.R.F. were supported by the DOE, BES, Scientific User Facilities Division.

\section{Author contributions}

Z.L. performed the sample synthesis with help of T.Z. Z.L. conducted the structural and basic physical property characterization with help of C.S., A.R. and T.Z.W.. E.S., E.G., R.D., C.S., A.R., Z.L. and J.F. performed XAS measurements. X.G. performed STEM characterization. Z.L., E.S., E.G., T.Z.W., T.C., M.R.F. and H.N.L. discussed the results. Z L. and H.N.L. designed the experiment and wrote the manuscript with inputs from all authors.

\section{Additional information}

Supplementary Information accompanies this paper at https://doi.org/10.1038/s41467019-08472-y.

Competing interests: The authors declare no competing interests.

Reprints and permission information is available online at http://npg.nature.com/ reprintsandpermissions/

Journal peer review information: Nature Communications thanks the anonymous reviewers for their contribution to the peer review of this work.

Publisher's note: Springer Nature remains neutral with regard to jurisdictional claims in published maps and institutional affiliations.

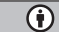

Open Access This article is licensed under a Creative Commons Attribution 4.0 International License, which permits use, sharing, adaptation, distribution and reproduction in any medium or format, as long as you give appropriate credit to the original author(s) and the source, provide a link to the Creative Commons license, and indicate if changes were made. The images or other third party material in this article are included in the article's Creative Commons license, unless indicated otherwise in a credit line to the material. If material is not included in the article's Creative Commons license and your intended use is not permitted by statutory regulation or exceeds the permitted use, you will need to obtain permission directly from the copyright holder. To view a copy of this license, visit http://creativecommons.org/ licenses/by/4.0/

(C) The Author(s) 2019 